durch die Vergabe von Forschungsdarlehen hat zwei wesentliche Aufgaben:

1. Übernahme des FuE-Risikos bei komplexen Aufgabenstellungen im Bereich von Schlüsselund Hochtechnologien durch den Staat und Gewährleistung eines effizienten und marktorientierten Technologietransfers;

2. Stärkung der Innovationskraft und Wettbewerbsfähigkeit der Unternehmen durch Abbau von Subventionen im Falle einer erfolgreichen Überführung von FuE-Ergebnissen.

Die Fördermittel werden als Forschungsdarlehen an die industriellen Verbundpartner vergeben. Grundfinanzierte Forschungsinstitute werden weiterhin direkt gefördert. Die Verbundpartner führen ein eigenständiges Controlling durch und entscheiden über die weitere Nutzung der von ihnen erzielten Ergebnisse.

Werden diese durch die beteiligten Unterneh- men nicht selbst genutzt, übernimmt der Staat das Darlehen, aber auch alle FuE-Resultate, die er durch einen kommerziell organisierten Technologietransfer weitergibt. Somit können sich für alle Beteiligten neue Chancen auf einen wettbewerblich strukturierten Technologietransfermarkt ergeben. Durch die Verbundpartner genutzte und überführte Ergebnisse sind das Resultat einer Forschung, bei der nur noch das Risiko durch den Staat mitgetragen wird. Die erfolgreiche Umsetzung der Forschungsergebnisse hat für den Industriepartner zur Konsequenz, daß das Forschungsdarlehen zu Konditionen, die denen des bereits existierenden BMFT- Programms »FuE-Darlehen für kleine Unternehmen zur Anwendung neuer Technologien« ähneln könnten, zurückgezahlt wird. Der Projektträger übernimmt eine neue Funktion. Er unterstützt die Integration außerdisziplinärer
Bereiche, die wesentlich zur erfolgreichen Überführung von FuE-Ergebnissen beitragen können, und fördert insbesondere kleine und mittlere Unternehmen beim Controlling und Technologietransfer.

Das neue Werkstofforschungsprogramm des BMFT folgt dem Leitbild »Werkstoffe für Schlüsseltechnologien«. Inwieweit sich Elemente einer »Integrierten Werstofforschung « zukünftig durchsetzen werden, bleibt abzuwarten. Ungeachtet der Profilierung des einen oder des anderen Leitbildes wird der Erfolg der Werkstofforschung zunehmend von den außerhalb der originären Werkstofforschung liegenden Bereichen entschieden. Dies gilt insbesondere für die frühzeitige Berücksichtigung ökologischer Belange und der rechtlichen Rahmenbedingungen.

Martin Socher, Bonn

\title{
(Umwelt-)Technik und zukunftsfähige Entwicklung
}

Produktion und Einsatz modernster Umwelttechnik werden in zunehmendem Maße von Politik und Wirtschaft als wichtige positive Faktoren sowohl für die ökologische als auch die wirtschaftliche Entwicklung gepriesen. Gleichzeitig wird die Umweltpolitik zu einem sehr kontrovers behandelten Element der aktuellen Standortdiskussion. In einer Vorstudie zu einem Projekt des Büros für Technikfolgenabschätzung des Deutschen Bundestags (TAB) wurden bisher zu dieser Thematik vorliegende Untersuchungen und Argumente zusammengetragen, analysiert und auf bestimmte Fragestellungen hin gebündelt und kommentiert.

\section{Umwelttechnik als Faktor für wirtschaftliche Entwicklung}

Bei der ökonomischen Betrachtung ist noch weitgehend unbestritten, daß mit der Umweltpolitik der 1970er und 1980er Jahre in der Bundesrepublik etwa aufgrund der Notwendigkeit des Einsatzes insbesondere sogenannter »nachgeschalteter Umwelttechnik « in der Gesamtbilanz - trotz auch eintretender Verdrängungseffekte - eine erhebliche Zahl von Arbeitsplätzen neu geschaffen worden ist. Angesichts der bisherigen Entwicklung des weltweiten Marktes für Umwelttechnologien und -dienstleistungen, der dortigen Position der Bundesrepublik sowie des übereinstimmend prognostizierten künftigen Wachstums in diesem Bereich bieten sich der bundesdeutschen Industrie ohne Zweifel positive Entwicklungschancen.

Die Industrie beklagt jedoch in der aktuellen Standortdiskussion nachdrücklich, daß für viele Branchen der Faktor Umweltschutzkosten sowie das »Regelungsdickicht« der deutschen Umweltpolitik (vor allem zu lange Genehmigungsverfahren) und die von ihr nicht ausreichend gewährleistete Planungssicherheit nachteilige Faktoren für den »Wirtschaftsstandort Deutschland « darstellten.

Im Hinblick auf das Kostenargument deuten die vorliegenden Untersuchungen demgegenüber darauf hin, daß selbst stark durch Umweltschutzkosten belastete Industriezweige in der
Vergangenheit kaum Veränderungen ihrer internationalen Wettbewerbsfähigkeit aufweisen. Gründe hierfür können in der generell vergleichsweise geringen Bedeutung der Umweltschutzkosten der deutschen Industrie bezogen auf den Produktionswert liegen (im Durchschnitt $0,7 \%$, bei den umweltkostenintensiven Zweigen 2,5\% mit zum Teil deutlich höheren Werten) oder auch in relativ geringen Preiselastizitäten der Nachfrage nach mit hohen Umweltkosten belasteten Produkten.

Untersuchungen dazu, inwieweit die recht pauschal geäußerte Umweltregulierungskritik zutreffend ist, existieren nicht. Feststellbar sind in vielen Fällen suboptimale ökonomische wie auch ökologische Anpassungsreaktionen von Unternehmen. Inwieweit diese - wie häufig behauptet - etwa auf die Dominanz ordnungsrechtlicher Instrumente in der Politik zurückzuführen sind, ist allerdings schwer abschätzbar. Mit Blick auf die ökologische Komponente wird von allen Seiten die wachsende Bedeutung und Notwendigkeit einer schon in die Produktionsprozesse bzw. die Produkte selbst einfließenden »integrierten Umwelttechnik « betont. Während die bislang vorwiegend eingesetzte nachgeschaltete Umwelttechnik in der Regel keine Material- oder Energieeinsparungen und häufig Verlagerungen von Umweltproblemen zwischen den einzelnen Medien Luft, Wasser und Boden bewirkt hat, verspricht die intergrierte Umwelttechnik eine höhere ökologische Effizienz, da sie an den eigentlichen Quellen von Umweltbelastungen, dem Stoff- und Energieeinsatz, ansetzt.

Zumindest mittel- und längerfristig kann sie auch ökonomisch bessere Perspektiven eröffnen, da etwa durch die Erhöhung der Ressourcenproduktivität auch die Gesamtproduktivität eines Produktionsprozesses verbessert werden könnte.

\section{Was kann Umwelttechnik leisten?}

Für eine genauere Bilanz in bezug auf den grundsätzlichen Stellenwert einer technik-bezogenen Umweltpolitik müssen verschiedene Bewertungskriterien herangezogen werden. Mit Blick auf die wirtschaftliche Frage zeigen die vorliegenden Untersuchungen, daß Umweltschutz für die betroffenen Unternehmen in der Regel Kosten bedeutet, allerdings selten in nachhaltig wettbewerbsverzerrender oder existenzgefährdender Höhe. Gleichwohl ist für eine Gesellschaft Umweltschutz zumindest auch insoweit nicht »umsonst « zu haben, als sie für die Finanzierung von Umweltschutzmaßnahmen auf die Befriedigung anderer Bedürfnisse oder möglicherweise "produktivere « Investitionen (z. B. im Bildungsbereich) verzichten muß.

Eine Betrachtung der ökologischen und insbesondere auch der internationalen Dimension des Einsatzes von Umwelttechnik umfaßt darüber hinaus Aspekte, deren Einbeziehung zu einer wesentlich kritischeren Einschätzung der Möglichkeiten und des Stellenwerts von Umwelttechnik angesichts der sich stellenden Probleme führt.

Mit dem Begriff des »sustainable development « kommt nun eine solche in verschiedener Hinsicht umfassendere Sichtweise erstmals in eine breitere öffentliche Diskussion. Er ist allerdings in den letzten Jahren in den wissenschaftlichen und den umwelt- bzw. entwicklungspolitischen Diskussionen letztlich nur zu einem vielver- 
wendeten Schlagwort geworden. Der Begriff ist bisher noch weitgehend auf dem Niveau einer abstrakten Idee verblieben, in zum Teil sehr verschiedener Weise definiert und interpretiert, je nachdem von wem bzw. in welchem Zusammenhang er reflektiert wird. Das Spektrum des Verständnisses von "sustainable development « reicht dabei von einer - weitgehend an ökonomischen und technologischen Aspekten ausgerichteten - ökologischen Modernisierung, wo allein auf dem Weg der Modernisierung des bestehenden Wachstums- und Zivilisationsmodells die (globalen) Probleme als lösbar erachtet werden, bis hin zu einer strukturellen Ökologisierung, wo weniger (ökonomische) Effizienzkriterien im Vordergrund stehen, als beispielsweise (globale) Verteilungsaspekte und insbesondere die Frage nach dem zukunftsfähigen bzw. langfristig aufrechterhaltbaren Produktionsund Konsumniveau einer Gesellschaft.

Neben der Berücksichtigung globaler Beziehungen und Ursache-Wirkungsfaktoren besteht ein wesentlicher Ansatz in dem letztgenannten, etwas weiter gefaßten Verständnis von »sustainable development « in der ganzheitlichen Einbeziehung von ökologischen, ökonomischen und sozialen Aspekten und Kriterien in Entwurf, Umsetzung und Bewertung von Entwicklungsstrategien bzw. einzelnen politischen Maßnahmen. Im Hinblick auf die ökologische Komponente stehen dabei zwei normative Zielsetzungen im Vordergrund:

- Die Nutzung von Rohstoffen auf ein Niveau zurückzuführen, das auch zukünftigen Generationen den gleichen Zugriff auf die Gesamtheit der verfügbaren Rohstoffressourcen gewährleistet bzw. das den Bestand an Rohstoffen nicht negativ verändert.

- Die Belastung der Umwelt durch Emissionen bzw. Reststoffe auf ein Niveau zu reduzieren, das die Verarbeitungskapazität der Ökosysteme für diese Einträge nicht überschreitet.

Es herrscht weitgehend Konsens darüber, daß gegenwärtig sowohl der Ressourcenverzehr bei den Rohstoffen als auch die Nutzung der Umwelt als Aufnahmemedium für Reststoffe deutlich über dem für eine (global) zukunftsfähige Entwicklung zuträglichen $\mathrm{Maß}$ liegen. Vor diesem Hintergrund wird im folgenden der Stellenwert einer an Umwelttechnik bzw. technischem Fortschritt orientierten Politik im Rahmen von »sustainable-development «-Strategien thesenhaft auf zwei Ebenen beleuchtet.

\section{Die nationale Ebene}

- Solange noch nicht annähernd ein gesellschaftlicher Konsens über Definition, Zielsetzungen und Operationalisierungsstrategien von "sustainable development « erzielt ist, scheint mir der Versuch, Technikpolitik an einer solchen gesellschaftlichen Leitidee ausrichten zu wollen, ein kurzsichtiges und sehr bald zum Scheitern verurteiltes Unterfangen zu sein.
- Ohne Zweifel wird ein Paradigmenwechsel in der Technikentwicklung und -politik unerläßlich sein. Für die Entwicklung, Einführung und Einsatzdauer einer neuen Technologie dürfen weniger rein ökonomische Kriterien (insbesondere das der Kostenreduktion) ausschlaggebend sein, sondern es muß eine Orientierung an Leitlinien wie »System- statt Einzelproblemlösungen«, »Minimierung von Stoff-, Energie- und Flächenverbrauch « oder »integrierte statt nachgeschaltete Technik « erfolgen.

Ein solcher Paradigmenwechsel ist jedoch vor allem aus zwei Gründen bei weitem nicht hinreichend für die Lösung der anstehenden Probleme:

a) Eine ökologische Optimierung der Technik kann letztlich immer nur spezifische Material-, Energie- oder Finanzeinsparungen je Produktionseinheit bewirken. Technik-orientierte Strategien können daher bestenfalls Gewinne auf Zeit darstellen, da für die ökologische Gesamtbilanz die Art bzw. Intensität der Nutzung von Techniken bzw. den erzeugten Produkten entscheidend sind. Es lassen sich zahlreiche Beispiele hierfür auflisten: Der positive Effekt von Pkw-Katalysatoren wird in dem Maße kompensiert, wie die Autofahrer häufiger ihr Fahrzeug benutzen und damit schneller und weitere Strecken fahren. Gleiches gilt für strom- und wassersparende Wasch- bzw. Geschirrspülmaschinen, wenn sich deren Benutzungshäufigkeit deutlich erhöht, oder für die sogenannten »Ökofernseher «, wenn sie dann in zwei- oder sogar dreifacher Ausfertigung in den Haushalten vorhanden sind.

b) Nicht selten sind Wirkungen im Zusammenhang mit der Herstellung und Nutzung von Technik nur unzureichend bekannt. Eingesetzte Stoffe können unvorhergesehene Probleme verursachen (Bsp. FCKW), ökologische, ökonomische oder soziale Sekundärwirkungen des Einsatzes bestimmter Stoffe bzw. Technologien sind häufig nur schwer oder gar nicht vorhersehbar.

Deshalb werden Strategien bzw. Maßnahmen auf nationaler wie internationaler Ebene erforderlich sein, die weit über die Denkkategorien der Technikentwicklung hinausgehen und insbesondere an zwei Punkten ansetzen müssen: Zum einen sind Veränderungen an einer Wirtschaftspolitik und -wissenschaft notwendig, die gesellschaftliche Rahmenbedingungen erheblich prägt und die sich bislang ausschließlich an Kriterien wie Globalisierung, Wachstum, Effizienz oder Gewinnmaximierung orientiert bzw, Wohlstand alleine über die Größe Bruttosozialprodukt definiert.

Zum anderen werden für verschiedene Bereiche spezifische Vermeidungsstrategien konzipiert und umgesetzt werden müssen, die an sozioökonomischen und sozialpsychologischen Fak- toren ansetzen und die die verschiedenartigen komplexen Ursache-Wirkungs-Mechanismen vieler Probleme berücksichtigen. Spätestens hier werden dann Fragen in bezug auf verschiedene gesellschaftliche Wertvorstellungen bzw. langfristig aufrechterhaltbare Lebensstile vor allem in den Industriestaaten zu stellen sein. In der Literatur wird die Frage, ob die für die Einleitung einer zukunftsfähigen Entwicklung notwendigen Reduzierungen von Ressourcennutzung und Reststoffanfall bei weiterem wirtschaftlichen Wachstum, gepaart mit hohem umwelttechnischen Fortschritt, realisiert werden können, sehr kontrovers diskutiert. Während sich z. B. Meadows et al. und Daly relativ pessimistisch äußern (Meadows et al. 1992; Daly 1992), geht die sogenannte Brundtland-Kommission in ihrem 1987 veröffentlichten Bericht »Unsere gemeinsame Zukunft « davon aus, daß eine global zukunftsfähige Entwicklung weiteres erhebliches wirtschaftliches Wachstum in den Entwicklungs- wie in den Industrieländern erfordert (World Commission on Environment and Development 1987, S. $50 \mathrm{ff}$ ).

In einem RWI/DIW-Gutachten von 1993 wird zu diesem Thema ausgeführt, daß eine gute gesamtwirtschaftliche Konjunktur bzw. ein stabiles Wachstum u. a. aus folgenden Gründen für eine reibungslose umweltbezogene Anpassung förderlich sei:

- »Im Zuge reger Investitionstätigkeit werden umweltbelastende Anlagen, Verfahren und Produkte schneller gegen moderne umweltschonendere ausgetauscht,

- Entwicklung und Diffusion moderner Querschnitts- und Schlüsseltechnologie beschleunigen sich (nicht zuletzt gespeist aus zunehmenden öffentlichen und privaten Forschungs- und Entwicklungsausgaben),

- die öffentlichen Hände sind bei entspannterer Finanzlage eher zu solchen Umweltinvestitionen bereit, die sich in gewissem Umfang zeitlich verschieben lassen (z. B. Sanierung der Kanalisation),

- die Konsumenten sind bei steigenden Einkommen und günstigen Einkommenserwartungen eher zu tatsächlichen Mehrausgaben für Umweltschutz bereit,

ökonomisch gesunde (kapitalkräftige) Unternehmen haben zudem größeren Spielraum hinsichtlich langfristiger und umfassender (integrierter) Umweltschutzmaßnahmen, während Unternehmen in finanziellen Schwierigkeiten eher gezwungen sein könnten, zur Erfüllung von Auflagen kurzfristigen, letztlich aber oft ineffektiven Umweltschutz zu betreiben « (RWI/DIW 1993, S. 13 ff.).

Gleichzeitig wird dort aber nicht verkannt, daß im Gefolge einer weiter zunehmenden Wirtschaftsaktivität die Umweltbelastung zunehmen kann. Zugleich wird darauf verwiesen, daß in wichtigen Wirtschaftsbereichen, so z. B. in der Elektrizitätswirtschaft, in der chemischen Industrie und der Stahlindustrie, zunehmende Wirtschaftsleistung bei abnehmender Umwelt- 
belastung realisiert werden konnte (RIW/DIW, 1993, S. 14). Es wird dann weiter argumentiert, daß somit der letztendliche Zusammenhang zwischen Wirtschaftswachstum, Anpassungslasten (und Umweltqualität, Hinzufügung des Verfassers) offen bleibe, daß aber auf längere Sicht - bei entsprechend ausgestalteter Umwelt- und FuE-Politik - zumindest kein unüberwindlicher Zielkonflikt zu unterstellen sei.

\section{Die internationale Ebene}

- Die Dimension der künftigen Herausforderung aus globaler Sicht läßt sich an einem relativ einfachen Beispiel verdeutlichen:

Bei einem für die nächsten 50 Jahre angenommenen Wachstum des BSP von $1 \%$ pro Jahr in den Industrieländern und 4\% in den Entwicklungsländern (also Werten, die eher am unteren Rand des bisher diesbezüglich Prognostizierten bzw. für wünschenswert Gehaltenen liegen) würde alleine schon die Zielsetzung einer Stabilisierung der heutigen Flußgrößen (d. h. der jeweils neu hinzukommenden Mengen) bei den Umweltbelastungen im rechnerischen globalen Durchschnitt eine Effizienzsteigerung um rund das Dreifache erforderlich machen. Dies entspräche im Weltdurchschnitt einer durch technischen Fortschritt zu induzierenden Senkung der Umweltbelastung pro BSP-Einheit um mehr als $2 \%$ pro Jahr. Ein Wert, den bis heute über einen längeren Zeitraum alleine Japan realisieren konnte.

Geht man davon aus, daß in vielen ökologischen Problembereichen aber zumindest eine Halbierung der Flußgrößen notwendig ist, wird eine Effizienzsteigerung um rund das Sechsfache bzw. eine jährliche Rate von mehr als 3,5\% erforderlich, was in der Tat schon Züge einer $» E f$ fizienzrevolution« tragen würde.

Bei Zugrundelegung der u.a. auch von der »Brundtland-Kommission « für notwendig erachteten Wachstumsraten von $2 \%$ pro Jahr in den Industrielstaaten bzw. $6 \%$ pro Jahr in den Entwicklungsländern würde entsprechend eine Effizienzsteigerung um das Siebenfache (bei Stabilisierung der Umweltbelastungen) bzw. um das Vierzehnfache (bei einer Halbierung), d.h. eine Effizienzsteigerungsrate von $4 \%$ bzw. $5,5 \%$ pro Jahr, notwendig werden.

Zieht man dann sinnvollerweise und auch realistischerweise noch in Betracht, daß erstens selbst eine Halbierung bestimmter Umweltbelastungen aufgrund der schon vorhandenen Schädigungs-Bestandsgrößen nach bisherigen Erkenntnissen nicht ausreichen wird (Bsp. $\mathrm{CO}_{2}$ ) und daß zweitens den Entwicklungsländern sicherlich derartige Reduktionen nicht zuzumuten bzw. sogar auf längere Zeit noch Steigerungen bei der Umweltbelastung zuzubilligen sind; ergäbe sich für die Industrielstaaten eine noch deutlich darüber hinausgehende Reduktionsnotwendigkeit.

Diese kann dann durchaus in dem Bereich einer »Umwelteffizienzsteigerung « um den Faktor 10 bis 50 liegen, wie er im Rahmen des niederlän- dischen Technologieprogramms »Sustainable Technological Development « für notwendig gehalten wird, um den Erfordernissen einer global zukunftsfähigen Entwicklung gerecht werden zu können (Vergragt/Jansen 1993, S. 136). Für den Verkehrsbereich wird hier z. B. die Notwendigkeit gesehen, ein Verkehrssystem zu realisieren, das die $\mathrm{CO}_{2}$-Emissionen um den Faktor 20 reduziert.

Spätestens derartige Größenordnungen liegen sehr wahrscheinlich jenseits all dessen, was mit technischen Lösungen - seien sie nachsorgender oder auch integrierter Natur - realisierbar sein wird. Um reale Verbesserungen erzielen zu können, müßte die Rate des technischen Fortschritts im Durchschnitt die Wachstumsrate des Bruttosozialprodukts mehr oder weniger deutlich überschreiten. Aufgrund verschiedener nicht außer Kraft setzbarer physikalischer Grenzen ist jedoch auch hier mit tendenziell abnehmenden Grenzerträgen zu rechnen. Deswegen erscheint eine dauerhafte Entkopplung zwischen Umweltverbrauch und erwirtschaftetem Sozialprodukt nicht realistisch. Ein sogenannter »technological fix «(d.h. die Vorstellung, die anstehenden Umweltprobleme seien allein durch technischen Fortschritt lösbar) ist somit sehr zu bezweifeln.

- Eine aus der Sicht der Industriestaaten erfolgreiche Politik, die zu spürbaren Reduktionen der Stoff- oder Energieverbräuche führen würde, hätte jedoch für viele rohstoffexportierende Entwicklungsländer nachteilige Effekte etwa in Form von - ceteris paribus - sinkenden Einnahmen. Dies würde umso schwerer wiegen, als zahlreiche dieser Länder finanziell ganz erheblich von diesen Exporten abhängig sind, die sich nicht selten auf die Ausfuhr von nur zwei oder drei Rohstoffen konzentrieren. Es wäre dann sicher sehr bald die Frage zu stellen, ob und in welcher Form bzw. Höhe Kompensationsmaßnahmen durch die Industriestaaten zu treffen wären.

All dies macht deutlich, daß eine Konzentration der technik-politischen Debatte auf nationale Aspekte - wie es momentan etwa in der bundesdeutschen Standortdiskussion geschieht angesichts der Notwendigkeit global zukunftsfähiger Entwicklungsstrategien eine unangemessene und letztlich wenig hilfreiche Verkürzung der Problembetrachtung darstellt. Um die genannten Dimensionen von Umweltverbesserungen auch nur näherungsweise erreichen zu können, sind konkret durch die Politik vorzugebende operationale Zielsetzungen notwendig, die etwa den Produzenten entsprechende verläßliche Planungs- und Kalkulationsgrundlagen bieten.

Das bisher einzige Beispiel für eine solche Strategie stellt der 1989 verabschiedete National Environmental Policy Plan « der Niederlande (N.E.P.P.) dar. In ihm werden z. B. zeitlich gestaffelte Zielvorgaben einer Reduktion der eutrophierenden Substanzen um $70-90 \%$ bis zum Jahr 2010 sowie eine Reduktion des Roh- stoffverbrauchs um $20-30 \%$ ebenfalls bis 2010 festgesetzt (Ministry of Housing, Physical Planning and Environment 1989).

\section{Fazit}

Die möglichen Potentiale nachgeschalteter und integrierter Umweltschutztechnik sind prinzipiell vor dem Hintergrund einer erhofften Entkopplung von Wirtschaftswachstum und Umweltbelastungen $\mathrm{zu}$ betrachten. Nachgeschaltete Umweltschutztechnik bedeutet in bezug auf die Umweltentlastung bei weiterem wirtschaftlichen Wachstum zumeist lediglich einen Gewinn auf Zeit, da ihre positiven Wirkungen bei weiter steigender Nutzungsintensität sehr rasch überkompensiert werden. Zudem sind in vielen Fällen Verlagerungseffekte zu konstatieren, d. h. Entlastungseffekte bei einem Umweltmedium führen zu zusätzlichen Umweltbelastungen in anderen Medien.

Integrierte Umwelttechnik bietet zwar grundsätzlich das Potential zur Entkopplung von Wirtschaftswachstum und Umweltbelastung. Dies setzt aber voraus, daß die Wachstumsraten der »Umweltproduktivität« im Durchschnitt langfristig über denen des BSP liegen müssen, was - wie bereits erwähnt - eher zu bezweifeln ist. Entgegen der zunehmend geäußerten hohen Erwartungen müssen technik-orientierte Politikstrategien angesichts der oben genannten Faktoren als grundsätzlich eingeschränkt bewertet und um andere, nicht-technische Ansätze zur Reduktion der aktuellen Umweltprobleme ergänzt werden.

Jürgen Kopfmüller, Karlsruhe

Literatur

- Daly, H. 1992: Steady-State Economics: Concepts, Questions, Policies, in: GAIA, Heft 6, S. 333-338

- Meadows, D. et al. 1992: Die neuen Grenzen des Wachstums, Stuttgart

- Ministry of Housing, Physical Planning and Environment 1989: National Environmental Policy Plan - To Choose or to Loose, The Hague

- RWI/DIW 1993: Umweltschutz und Industriestandort. Forschungsbericht 10103 62, UBA-FB 93031, UBA-Bericht 1/1993, Berlin

- Vergragt, P./Jansen, L. 1993: Sustainable Technological Development: The Making of a Dutch LongTerm Oriented Technology Programme, in: Project Appraisal, Vol. 8, No. 3, S. 134-140

- World Commission on environment and Development 1987: Our Common Future, Oxford/New York

\section{Redaktionsschluß der nächsten Ausgabe:}

2. Dezember 1994 
(c) 20I0 Authors; licensee IÖW and oekom verlag. This is an article distributed under the terms of the Creative Commons Attribution Non-Commercial No Derivates License (http://creativecommons.org/licenses/by-nc-nd/3.o/), which permits unrestricted use, distribution, and reproduction in any medium, provided the original work is properly cited. 